\title{
VOCABLOS DIALECTALES RELACIONADOS CON LA NATURALEZA DEL TERRENO
}

\author{
María Pilar Garcés \\ Universidad de Málaga
}

Nuestro trabajo se centra en el análisis de las voces dialectales que se emplean en la zona pirenaica para designar diversos aspectos de la topografía y de la naturaleza del terreno.

El objetivo es determinar el arraigo y la extensión geográfica actual de estos términos poco conocidos en la lengua normativa, ya que en su mayoría se consideran dialectalismos. Como se trata de una parcela léxica no sometida a la necesidad de nueva denominación que impone el desarrollo histórico, cultural y técnico de un pueblo, sino que, por el contrario, es un vocabulario de carácter tradicional muy arraigado en el terruño, son muy numerosas las palabras mantenidas en el habla viva.

En muchos casos encontramos varios términos dialectales para expresar un mismo sentido, por ello, vamos a partir del significado para estudiar los distintos significantes que lo actualizan, lo que nos permitirá además dar una visión de conjunto de las voces patrimoniales preferidas por los hablantes frente a las de la lengua oficial en este campo significativo ${ }^{1}$.

1 Los materiales que nos han servido de base para nuestro trabajo son los mapas contenidos en el Atlas lingüístico y etnográfico de Aragón, Navarra y Rioja publicado por M. Alvar con la colaboración de A. Llorente, T. Buesa y E. Alvar. Madrid, 1979-83, 12 vols., los mapas estudiados corresponden al vol. IX, n. ${ }^{\text {w }} 1347-1404$. 


\section{1. 'RIBAZO'}

Espuenda se registra en puntos de Huesca (Hu 101, 400, 403, 406), la variante espuena, con simplificación del grupo - ND-, en Hu 201, 207; la forma espona, que se corresponde con el vocablo catalán, se documenta en puntos de la frontera oriental (Hu 205, 402); en el norte y centro de Navarra las voces ezpuenda y ezpondón son las más generales (Iribarren, 243); en Na 103 no se establece ninguna diferencia significativa entre primitivo y derivado, en otros puntos se hace una distinción basada en el sema 'tamaño': ezpondón 'ribazo de mayores proporciones'/ ezpuenda 'ribazo de proporciones más pequeñas' en $\mathrm{Na} 400,401$, 402; en otros casos la variación viene dada por la distinta localización geográfica: ezpuenda ( $\mathrm{Na} 100,202,205,403)$, espuenda (Na 106, 201,

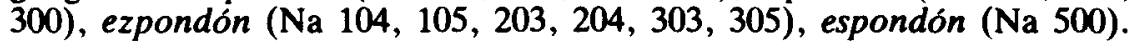

Esta misma forma la hallamos además para designar 'ribazo en pendiente' en algunas localidades y 'límite entre dos fincas', en otras ${ }^{2}$, por lo que en varios puntos se ha producido una neutralización de semas diferenciadores y espuenda actualiza los tres significados en $\mathrm{Na} 201,300$; ezpuenda en $\mathrm{Na} 202$, 403, espuena en $\mathrm{Hu} 201$ y ezpondón en $\mathrm{Na} 305$ designan 'ribazo' y 'ribazo en pendiente'; en Hu 403 se establece una distinción significativa entre espuenda 'ribazo' y esponal 'ribazo en pendiente'.

EI DRAE registra la voz espuenda como palabra regional aragonesa y navarra con el significado de 'borde de un canal o de un campo' (s.v.), pero no recoge la variante ezponda, que es la más usual en Navarra.

Es un vocablo derivado del latín s p o n d a 'armazón de cama', que en romance tomó la acepción de 'borde de cama' y de ahí, por un desplazamiento semántico las de 'inclinación del terreno', 'ribazo', 'margen', que son las que también tienen el catalán espona (DCVB), el gascón espoune, espounde, espoundre (Palay, 461) y el vasco esponda (Azkue), donde es un préstamo procedente del romance aragonés.

La palabra ripa en $\mathrm{Hu} 102,105$ y la derivada ripazo en $\mathrm{Hu} 108,109$, $202,403,407,600,601$ son voces patrimoniales aragonesas tal como nos muestra el mantenimiento de la oclusiva sorda intervocálica (< latín r i p a 'orilla', 'margen de un río'), mientras que en el resto de este ámbito lingüístico la forma más generalizada es la propiamente castellana ribazo.

Con el significado de 'ribazo' la forma marguin y el derivado marguinazo se documentan en Huesca y en el norte y centro de Zaragoza; en general, el uso del primitivo o del derivado responde a una localiza-

\footnotetext{
${ }^{2}$ Cfr. ALEANR, mapas $23,25 *$
} 
ción geográfica distinta; sólo en Hu 112 se establece una diferencia significativa entre marguinazo 'ribazo grande' / marguin 'ribazo pequeño'. En algunos casos encontramos un ensordecimiento de la consonante oclusiva: marquin ( $\mathrm{Hu} 204$ ), marquinazo (Hu 106, 203), lo que podría explicarse por un cruce ente marguin y el nombre marca o la forma verbal marcar 'señalar con marcas o límites', ya que estas denominaciones expresan contenidos que están dentro del mismo campo significativo, o bien por un caso de ultracorrección.

Con el sentido de 'limite entre dos fincas' 3 se documenta el vocablo marguin en el norte y parte occidental de Huesca, en el norte y zona

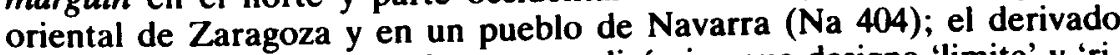
marguinazo en $Z$ 401 , donde es voz polisémica que designa 'limite' y 'ribazo' al haberse producido la neutralización de semas diferenciadores, lo mismo que ha ocurrido en $\mathrm{Hu} 603, \mathrm{Z} 100,300$ con el primitivo marguin.

La forma dialectal aragonesa está emparentada con la voz castellana margen 'extremidad y orilla de una cosa' (DRAE) con el vasco margin 'límite' (Azkue), y el catalán marge 'vora' (DCVB). Todas ellas remontan al latín m a r g o, - i n i s 'borde', 'margen'; el término normativo supone una evolución de tipo culto, pero en castellano también se documentan representantes hereditarios como muestra la existencia de la forma marcen 'faja de tierra que se señala para la siembra' en Teruel, en la parte occidental de Rioja y Navarra y en varios pueblos de Castilla (DEEH) ${ }^{4}$.

El aragonés marguin parece ser una forma popular, aunque presenta una evolución fonética anómala. V. García de Diego supone un influjo del nominativo $\mathrm{m}$ a $\mathrm{r}$ g $\mathrm{o}^{5}$; sin embargo, lo más probable es que este término llegara al romance aragonés a través del antiguo vasco local, donde los préstamos antiguos del latín mantienen la articulación oclusiva $[\mathrm{g}]$ en estos grupos ${ }^{6}$.

\section{2. 'ATAJO'}

Alcorce es la forma dialectal que se registra en Huesca, excepto en la frontera oriental donde se documenta el término catalán dressera, endressera (DCVB), en el norte, centro y sureste de Zarago-

3 Cfr. ALEANR, mapa 23.

4 Cfr. ALEANR, mapa 38.
V. García de Diego, Contribución al diccionario hispánico etimológico. Madrid,

6 H. Lausberg, Lingüistica románica. Madrid, 1976, vol. I, p. 322, nota 14. 
za y en Navarra. Es un sustantivo postverbal de alcorzar 'acortar', derivado del latín vulgar $\mathrm{c}$ ŭ $\mathrm{r} \mathrm{t}$ i a $\mathrm{r}$ e, más exactamente de una variante *a c c u r t i a r e (DCECH, III, 216) ${ }^{7}$; en algunos puntos encontramos una forma diptongada alcuerce (Hu 300, 302, 304, Z 300, 400, 402, $604)^{8}$, alcurce en $\mathrm{Hu} 202$, quizá por influencia de la palabra cruce, muy próxima tanto semántica como fonéticamente.

En algunos casos conviven como sinónimos el término dialectal y el de la lengua general atajo ( $\mathrm{Na} 202,204,400,502, \mathrm{Hu} 104,106, \mathrm{Z} 301$, $302,305)$; en un pueblo de Navarra ( $\mathrm{Na} \mathrm{103)} \mathrm{la} \mathrm{voz} \mathrm{dialectal} \mathrm{y} \mathrm{el} \mathrm{voca-}$ blo vasco correspondiente lasterbidia (Azkue); en Na 200 las tres voces conjuntamente: atajo, alcorce, lasterbidea.

Por su relación con el adjetivo corto aparecen las variantes acorzo (Lo 602), alcorzo ( $\mathrm{Na} \mathrm{500).}$

El término dialectal trestallo tiene una extensión mucho menor. En Hu 101 los dos vocablos dialectales se consideran sinónimos; en $\mathrm{Hu} 112$ se establece una diferencia significativa entre alcorce 'atajo' / trestallo 'atajo con mal piso'; en otros dos casos trastajo ( $\mathrm{Hu} 111$ ), trestajo (Z 202) se registran como única denominación.

\section{3. 'UMBRIA'}

Para designar el 'lado de un monte donde no da el sol' encontramos dos formas procedentes de la base latina o p ā c u s 'sombrío': paco, que se extiende por la parte occidental de Huesca, puntos de Zaragoza

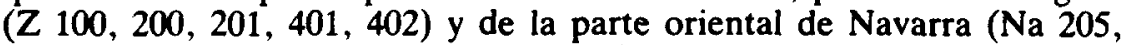
$400,401,402,403$ ); las voces ubago (Hu 201, 202, 403), obago (Hu 200, 204, 401, 406), obac (Hu 402), obaga (Hu 205, 404, 408), que se registran en el norte y centro oriental de Huesca y están emparentadas con el catalán obaga (DCVB), y con las gasconas ubàc (Lavedan), ubàch, uboùch (Gers) (Palay, 999), que tienen este mismo significado.

Los derivados pocino (Hu 106, 203, 204, 206), pacino (Hu 405) proceden de una hipotética base *o p à c i n u s, mientras que el derivado paquizo (Hu 100,110,112), que se relaciona a su vez con el gascón pakís (N. Lescun) ${ }^{9}$, remonta a una base *o p a c i c i u, ambos étimos tendrían el mismo valor que el adjetivo primario.

${ }^{7}$ M. Alvar señala que en esta palabra más que hablar de epéntesis, tal como sucede en otros casos en aragonés, hay que pensar en una confusión de prefijos, vid. El dialecto aragonés. Madrid, 1953, p. 203.

8 Como es sabido, las vocales cerradas pueden diptongar en aragonés, vid. M. Alvar, op. cit., p. 153.

${ }^{9}$ Cfr. W. D. Elcock, De quelques affinités phonetiques entre l'aragonais et le béarnais. París, 1938, mapa 16. 


\section{4. 'CERRO'}

La voz tozal con el significado de 'pequeña montaña que acaba en punta' se documenta en Huesca, en puntos de Zaragoza $(Z 601,605)$ y de Teruel (Te 204); la correspondiente forma catalana tossal aparece en varias localidades fronterizas ( $Z$ 606, Te 202, 207).

En algunos casos se han neutralizado los semas diferenciadores de 'cerro' y 'cerro cónico' y ambos significados son actualizados por esta misma palabra en $\mathrm{Hu} 100,106,108,206,207,300,302,403,601$, $\mathrm{Z}$ 605, Te 204; en otros puntos se establece la distinción de los dos valores mediante procedimientos morfológicos: empleo de sufijos tozal / tozalico (Hu 301), tozal / tozalet (Hu 603), o de determinantes tozal / tozal punchudo (Hu 204) para actualizar los sentidos de 'cerro' y 'cerro cónico' respectivamente.

Con el significado específico de 'cerro cónico' encontramos tozal en $\mathrm{Hu} 405$ y tozalón en Hu 110, mientras que para 'cerro' se utilizan formas procedentes de étimos distintos: plana y corona en cada caso.

La denominación tozal también se documenta para designar 'cresta rocosa', pero en todos los casos va acompañada de un determinante para diferenciarla de los otros sentidos: tozal 'cerro', y 'cerro cónico' / tozal de roca 'cresta rocosa' en Hu 302; tozal 'cerro cónico' / tozal pedregoso 'cresta rocosa' en $\mathrm{Hu} 405$.

Corominas supone que el primitivo toza es una palabra antigua, de origen incierto, aunque probablemente remonte a una base ${ }^{*} \mathrm{t}$ a u c i a 'mata, cepa de árbol', cuyo significado original sería 'cepa' o bien 'raigambre', 'mata' de donde evolucionó a 'objeto abultado', 'cogote abultado' y finalmente 'cogote' (DCECH, V, s.v. tozuelo) ${ }^{10}$, el paso semántico de 'cerviz' a 'montaña' se debe a un proceso metafórico. $V$. García de Diego señala una base latina t o n s u s 'pelado', de donde se pasaría al sentido de 'cabeza' y de éste al de 'cabezo' o 'monte' a través de una comparación metafórica (D.E.E.H.); pero resulta difícil explicar el paso del grupo latino - NS - al fonema interdental $/ \theta /$ del aragonés y de otros derivados españoles como tozuelo, tozudo, etc.

\section{5. 'CRESTA ROCOSA'}

La voz ralla se documenta en el norte y centro de Zaragoza y en puntos del norte y centro occidental de Huesca.

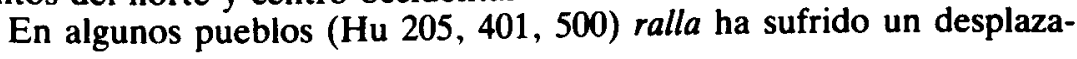

1" De este mismo origen es también el vocablo aragonés tozar 'embestirse, darse golpes con la cabeza los animales', que se registra en Huesca y en la parte oriental de Zaragoza y de Teruel; cfr. $A L E A N R$, IV, mapa 604. 
miento semántico y designa 'ladera cubierta de grandes piedras', mientras que para el significado de 'cresta rocosa' se utilizan palabras pertenecientes a étimos distintos: canteres en $\mathrm{Hu} 205$, pedregal en $\mathrm{Hu} \mathrm{401,}$ piedras en $\mathrm{Hu} 500$; en otros dos casos ralla se mantiene con el valor de 'cresta rocosa' y el sintagma subida con ralla en $\mathrm{Hu} 110$, o el derivado rallera en $\mathrm{Hu} 111$ hacen referencia a una 'ladera cubierta con grandes piedras'.

Es una forma procedente del latín r a d ŭ 1 a 'raedera' (DCECH, IV, 761); de origen iberorromance son las voces que se documentan en gascón: arralhe 'éboulis de pierres', arralho 'grosse pierre qui se détache d'un rocher' ${ }^{11}$ y en vasco: arrailu 'hendidura', arraildu 'hender' (Azkue).

Otro término dialectal para actualizar este significado es cingla (Hu 102), cinglo, cinglos en puntos de la frontera oriental del sur de Huesca, de Zaragoza y en sitios aislados de Teruel (Te 300, 501, 504, 601); en $\mathrm{Te} 504$ se establece una diferencia significativa entre cinglo 'cresta rocosa por donde no se puede pasar' y el derivado cinglaja 'cresta rocosa por donde se puede pasar'.

Esta palabra la encontramos además con otros significados que están dentro de este mismo campo semántico: cingla en $\mathrm{Hu} 101,104, \mathrm{Na}$ 401 designa 'saliente de una roca empleado como abrigo'; cinglo en $\mathrm{Hu}$ 110 es 'roca o ralla de unos quinientos metros'; cingla en Z 202 denomina la 'roca pegada a otra que tiene una grieta'. En Hu 102 cingla y en Hu 601 cinglo es palabra polisémica que cubre los significados de 'cresta rocosa' y 'saliente de una roca empleado como abrigo'; en Te 501 se diferencia léxicamente cada uno de los significados mediante la utilización de la forma simple y de un sintagma: cinglo 'cresta rocosa' / cueva en un cinglo 'saliente de una roca usado como refugio'.

Es una voz derivada del latín c ì n g ù l u m 'cinturón', que se corresponde con el castellano cincho, cincha, con el catalán cingle (DCVB), el gascón sinclo 'corniche, gradin herbeux dans les rochers escarpés' (Palay, 907) y el vasco zingla 'cincha' (Azkue). Corominas supone que el paso semántico de 'cinturón' a 'cresta rocosa' se explica porque, en principio, esta designación se aplicaría a una "mole ceñida de rocas o de crestas rocosas', y después ampliaría su significado para designar 'cresta rocosa' en general (Diccionari, II, 710-2).

Otros vocablos dialectales sólo documentados en una o dos localidades: catarra (Hu 100), el derivado catarreras (Hu 204), cantarera (Hu 206), penna ${ }^{12}$ (Hu 200), tartera (Hu 201).

"Cfr. G. Rohlfs, Le Gascon. Éludes de philologie pyrénéenne, $3^{\mathrm{eme}}$ ed. Tübingen, 1977, pp. 73-74.

i2 Cfr. A. Badía, El habla del valle de Bielsa. Barcelona, 1950, p. 314 recoge penna 


\section{6. 'DESPRENDIMIENTO'}

Se registran dos formas procedentes de un mismo étimo: lurte, alurte (Hu 101), lurta ( $\mathrm{Na} 205,206,401)$ en la parte occidental de Huesca y oriental de Navarra; liz, esliz en la zona nordoriental de Huesca.

Estas voces, junto con la variante alud, que es la que se ha extendido a la lengua general, derivan de un tipo pre-ibérico y pre-vasco ${ }^{*} I \bar{u} \mathrm{t}$ e, ${ }^{*} \mathrm{I}$ ù $\mathrm{t} \mathrm{a}$, conservado en algunos puntos de los Pirineos occidentales y de los Alpes, con una variante dialectal *l i t e, propia de los $\mathrm{Pi}$ rineos centrales; en los lugares más vasquizantes (Bearn, Navarra y valles aragoneses adyacentes a este ámbito lingüístico) se produjo un cruce con el vasco elur 'nieve', lur 'tierra' (Azkue), que originó el tipo lur(te); por otra parte, la base ${ }^{*} 1$ i t e que ha dado lugar a las formas altoaragonesas liz, esliz, las gasconas lit, eslit (Palay, 621) y al catalán llido, puede haber surgido por el influjo de una hipotética raíz ${ }^{*} l$ i $t \mathrm{u}$, de origen sorotáptico, con un significado como 'desbordamiento, avalancha' que se corresponde perfectamente con el sentido de 'desprendimiento de tierra o nieve' que tienen las palabras romances (Diccionari, $\mathrm{V}$, 204-6) ${ }^{13}$

\section{7. 'GLERA'}

La denominación glera con el significado específico de 'piedra de distintas formas y tamaños que hay a la orilla del mar o en los barrancos' es general en Huesca, junto con la variante llera en el norte y centro oriental, con' una palatalización del grupo consonántico inicial característica de esta zona ${ }^{14}$; en catalán occidental la variante más conocida es glera (Diccionari, IV, 525-6). Es una palabra procedente del latín g 1 a r è a 'cascajar, arenal'.

En varios puntos del dominio lingüístico aragonés esta forma se registra con otros sentidos, lo que supone que, en algunas localidades, se ha convertido en voz polisémica que actualiza varios significados: gelera, con anaptixis, en Hu 204, glera en Hu 206 y llera en Hu 207 desig-

con el valor de 'peña, piedra muy grande'; la geminación de la nasal alveolar es un fenomeno muy frecuente en belsetán.

$13 \mathrm{Vid}$. T. Buesa, «Léxico vasco relativo al tiempo en la Navarra nordoriental (Partido de Aóiz)», en Homenaje a F. Induráin. Zaragoza, 1972, pp. 92-94; vid. además, X. Ravier, «Le theme LUR dans le lexique gascon», en Festschrift für J. Hubschmid. Bern, 1982, pp. 937-952.

14 Cfr. J. Saroïhandy, «El català del Pirineu, a la ralla d'Aragó», en Primer Congrés Internacional de la llengua catalana (1906). Barcelona, 1908, pp. 331-334. 
nan conjuntamente 'glera', 'ladera cubierta de grandes piedras' y 'sitio por donde corre el agua cuando ha llovido mucho'; en dos casos (Hu 200, 202) lera denomina específicamente 'ladera cubierta de grandes piedras', mientras que la voz dialectal ronal ${ }^{15}$ en Hu 202 y cantal en $\mathrm{Hu} 200$ designan 'glera'.

En Hu 108 glera, en Hu 402 llerall actualizan los significados de 'glera' y 'lecho por donde corren las aguas pluviales'; en otros puntos la forma estudiada se ha hecho específica para 'rambla' y se emplean voces procedentes de étimos diferentes para 'glera': cascarral / gelera (Z 200), bolos / glera (Hu 100), espedregal / glera (Hu 112), graba / llera (Hu 302), rollizos / llera (Hu 403); en otros casos la distinción significativa se mantiene a través de diversas especificaciones: piedra de glera / glera (Hu 106), ruellos de glera / glera (Hu 203), o de sufijos derivativos: llera / llerall (Hu 404), para designar los valores de 'glera' y 'rambla' respectivamente.

Otros términos dialectales, que no son específicos para este significado, sino que tienen además otros sentidos son ruejo en $\mathrm{Na} 405$, donde designa 'glera' y 'canto rodado'; ruello en $\mathrm{Hu} 101, \mathrm{Na} 403$ y bolo en $\mathrm{Hu} 100$, que expresan 'glera' y 'piedra redonda de gran tamaño'.

\section{8. 'PIEDRA PEQUeÑa'}

La denominación zaborro, zaborra se extiende por Huesca, puntos de Zaragoza y de la zona occidental de Navarra. En general la variación de género responde a una distinta localización geográfica ${ }^{16}$; sóle en un caso ( $\mathrm{Hu} \mathrm{108)} \mathrm{se} \mathrm{establece} \mathrm{una} \mathrm{diferencia} \mathrm{significativa} \mathrm{entre} z a$ borra 'piedra de tamaño mayor' / zaborro 'piedra de tamaño menor'.

En otros casos en los que el sema 'tamaño' es relevante, la distinción se establece a través de formas procedentes de étimos distintos: piedra / zaborra (Hu 110), piedreta / zaborra (Hu 111), 'guijarro pequeño' y 'guijarro grande' respectivamente; por el contrario zaborra de-

15 Esta palabra está emparentada con el catalán runa 'escombros de piedras y arena' (DCVB); según Corominas, el término catalán procede del latín $r$ ũ $d$ e $r$ a 'escombros, ripio', plural de $r \bar{u} d u s,-\varepsilon r$ i s, con una disimilación de las vibrantes, dando * r ù d è n a, cuya evolución a runa es normal; para la forma aragonesa rona señala un étimo $\mathrm{r}$ a u d e $\mathrm{r}$ a ("r a u d e $\mathrm{n}$ a ), pues consta que $\mathrm{r}$ u $\mathrm{d}$ u s se empleaba vulgarmente confundido con r a u d u s, - $\mathrm{e}$ r i s 'piedra en bruto', 'lingote sin trabajar' (DCECH, II, 638-40).

16 No es por tanto válida la distinción significativa que se hace en el DRAE entre ambas voces dialectales: zaborra 'piedra pequeña', zaborro 'cascote de yeso, yesón' (s.v.). 
signa 'guijarro pequeño' en Hu 112 frente al cantal 'guijarro grande', e igual distinción se da en $\mathrm{Na} 401$ entre zaborra / piedra.

En algunos pueblos ( $\mathrm{Na} \mathrm{404,} \mathrm{Hu} \mathrm{106,} \mathrm{202,} \mathrm{Z} \mathrm{100)} \mathrm{esta} \mathrm{forma} \mathrm{se} \mathrm{ha}$ especializado con el valor de 'china, piedrecilla pequeña generalmente dura y blanquecina'; sin embargo, el interés del hablante por precisar que se trata de una piedrecita de reducida dimensión, muy inferior a la normal, ha supuesto que, a veces, se precise esta característica a través de los diminutivos o de los determinantes: zaborra pequeña ( $\mathrm{Na} 405$ ), zaborreta (Hu 100), zaborrica (Z 400).

Es un vocablo derivado del latín $s$ a b ŭ $r$ r a 'lastre', 'arena', que tiene correspondencias en el catalán antiguo saorra, hoy sorra (DCVB), y en el vasco zabor 'piedras pequeñas con que se hace la argamasa', 'escombros, residuos' (Azkue). Del aragonés ha pasado al murciano, donde se registra zaborra 'piedra pequeña' ${ }^{17}$.

Otros términos dialectales documentados son: ruejo 'piedra pequeña' en $\mathrm{Na} 601, \mathrm{Z} 201$; en $\mathrm{Na} 309,602$ ruejo designa 'piedra pequeña' y 'canto rodado'; en Z 100 se establece una distinción significativa entre ambos significados a través del determinante: ruejo menudo 'piedra pequeña' / ruejo 'canto rodado'; sólo en un punto (Hu 102) hallamos tantonico 'piedra pequeña' y 'canto rodado' (Rohlfs), y ragualla en Hu 201 con el valor de 'piedra pequeña'.

\section{9. 'CANTO RODADO'}

No encontramos formas exclusivas para designar 'canto rodado', los términos documentados los hemos registrado anteriormente para expresar otros contenidos.

Ruello, o con adaptación a la fonética castellana ruejo, se recoge en diversos puntos de Navarra, del norte y centro de Zaragoza y del norte de Huesca; son descendientes del latín $r \quad \delta$ t u $l$ u s 'ruedecita', a través de una comparación metafórica fácilmente identificable (DEEH).

El otro vocablo es glera que, en unos casos, aparece con distinto género: glero $(\mathrm{Z} 600,601,603)$, o con sufijos derivativos: glerizo (Hu 601), glericete ( $\mathrm{Hu} 600)$, aunque en ningún punto esta variación sirva para establecer diferencias significativas, ya que para designar 'glera' se utilizan formas procedentes de étimos distintos; en otras ocasiones aparece como elemento determinante de un nombre de significado más amplio: piedra de glera (Hu 103, 105, 107, 109, 110, 204),

${ }^{17}$ Cfr. J. Garcia Soriano, Vocabulario del dialecro murciano, (ed. Facsímil de la 1." ed. Madrid, 1932). Murcia, 1980, p. 134. 
ruello de llera (Hu 207), frente a la denominación glera, llera que designa el 'conjunto de piedras de diversas formas y tamaños'.

\section{0 'Arcilla'. 'GredA'}

En las denominaciones de 'arcilla' y 'greda' hallamos una forma común para ambas, buro, que, en algunos puntos, designa indistintamente los dos tipos de tierra al haberse producido una neutralización de los semas diferenciadores: buro en $\mathrm{Na} 205, \mathrm{Hu} 400, \mathrm{Z} \mathrm{300,303,600,} \mathrm{y} \mathrm{el}$ sintagma tierra buro en Hu 603; esto se explica por el hecho de que ambas clases de tierra son muy semejantes por sus propiedades $y$, además, presentan distintos matices cromáticos que explican las posibles confusiones.

En algunos puntos se intenta mantener la distinción a través de los determinantes: buro / buro blanco ( $\mathrm{Na} \mathrm{602),} \mathrm{buro} \mathrm{/} \mathrm{tierra} \mathrm{burenca}$ (Hu 102), buro colorau / buro (Hu 110), buro royo / buro gris (Hu 203), buro rojo / buro blanco (Hu 305), buro rojo / tierra de buro blanco ( $\mathrm{Hu} 600)$, buro rojo / buro ( $\mathrm{Hu} 601)$, buro / buro colorao ( $\mathrm{Z}$ $100)$, buro colorau / buro ( $Z$ 401), que designan 'arcilla' y 'greda' respectivamente.

En las restantes localidades en las que se documenta la palabra buro, ésta se ha convertido en voz específica para expresar 'greda' y se emplea para 'arcilla' el término de la lengua oficial: arcilla ( $\mathrm{Na} \mathrm{206,}$ $402, \mathrm{Z} \mathrm{200}$ ), la variante dialectal arguila ( $\mathrm{Hu} \mathrm{401),} \mathrm{o} \mathrm{una} \mathrm{denominación}$ de significado general que va determinada por un adjetivo o un complemento: tierra roya ( $\mathrm{Na} 403,404, \mathrm{Hu} 202,204,206, \mathrm{Z} 201,601$ ), tierra delgada ( $\mathrm{Na} 600)$, tierra fuerte (Hu 303, 403), tierra de saso ${ }^{18}$ (Hu 304).

A veces buro se registra con un significado más específico: buro 'ar-

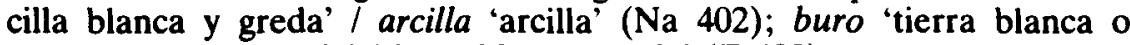
roya que no cria nada' / buro blanco 'greda' ( $Z 400)$.

Respecto a su origen etimológico se han propuesto dos hipótesis. V. García de Diego propone una base b u $t$ y $r$ u $m$ 'manteca', del griego ßov́zugov, quizá por el color, o por la consistencia del barro arcilloso semejante a la de la manteca (DEEH). Corominas cree que es un préstamo del francés bure 'paño buriel' o del occitano buro 'id', derivados regresivos de burel, de origen desconocido, pero posiblemente emparentados con el adjetivo de creación romance *b u $r$ i u s 'oscuro' (DCECH, I, 700-1).

${ }^{18}$ Cfr. M. Peralta, Ensayo de un diccionario aragonés-castellano (Zaragoza, 1836), ed. facsímil publicada en Madrid, 1984, p. 29: saso 'tierra ligera y opuesta á la vega'. 


\section{1. 'BARRO'}

La forma dialectal aragonesa bardo es general en Huesca, frente al resto del dominio lingüístico donde se registra la voz castellana barro. Este vocablo tiene correspondencias en gascón: bard, -de 'bove, limon, bauge, terre détrempée pour faire le torchis' (Palay, 103), pero es casi desconocido en catalán. Esta extensión geográfica y la alternancia -rr-, -rd- indican que es una voz prerromana, quizá más celtibérica que ibérica (DCECH, I, 528-9) ${ }^{19}$.

En algunos puntos el término dialectal y el normativo se usan indistintamente (Hu 103,105, 107, 110,111, 407, Z 200, 202), no estableciéndose ninguna diferencia significativa entre ellos.

Para designar el 'sitio donde se hace mucho barro' se utiliza la forma dialectal aragonesa en las localidades señaladas, a la que se añaden distintos sufijos derivativos ${ }^{20}$; por lo común, se mantiene la corres-

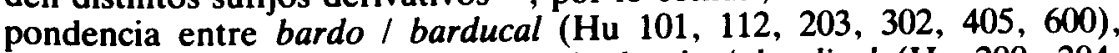
bardo / bardera (Hu 104, 108, 201), bardo / bardizal (Hu 200, 204, 207), bardo / barduscal (Hu 300). En los casos en los que aparecen como sinónimos barro y bardo, en la derivación se producen los siguientes hechos: a) se prefiere emplear la base dialectal: barducal (Hu 103, 105, 111), bardizal ( $Z$ 200); b) alternan los dos radicales: barrizal I bardera (Hu 107), barrizal / bardizal (Hu 110); c) se recurre a un sintagma en el que se integran las dos denominaciones simples: hay mucho barro / bardo ( $\mathrm{Hu} \mathrm{407);} \mathrm{d)} \mathrm{se} \mathrm{utiliza} \mathrm{un} \mathrm{término} \mathrm{dialectal} \mathrm{de} \mathrm{origen} \mathrm{dis-}$ tinto: entrasca (Z 202).

En dos localidades del centro oriental de Huesca (Hu 401, 406) se emplea el préstamo catalán fango; sin embargo, la voz dialectal barducal es la que designa el 'sitio lleno de barro'.

\section{2. 'LAgunA'}

La denominación ibón 'laguna' es una de las voces que con más frecuencia se utiliza para caracterizar el «vocabulario pirenaico». En el DRAE se señala que este regionalismo aragonés tiene el significado de 'lago de los Pirineos de Aragón' (s.v.); sin embargo, éste no es su único

$19 \mathrm{~V}$. García de Diego propone una base céltica "b a $r$ r i t o s 'lodo' (DEEH).

20 La mayoría de estos vocablos derivados presenta la unión de varios elementos: los sufijos -izo (>-i c i u) o -uco (>-u c (c) u) ambos de carácter peyorativo; y el sufijo -al (>-a 1 i s) que indica 'lugar': vid. G. Rohlfs, «Los sufijos en los dialectos pirenaicos», en Pirineos, VII, 1951, pp. 467-525. 
sentido y, además, tampoco es una palabra extendida por todo el dominio lingǘstico, ya que su empleo se reduce a unos cuantos pueblos al-

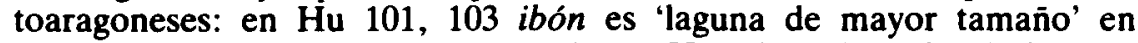

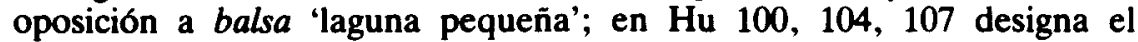
'lago pirenaico originado por una corriente subterránea de agua'; en Hu 300 denomina la 'laguna en la que nace el agua'; en Z 101 el 'terreno pantanoso'; en los demás casos en los que se documenta (Hu 102, $106,203, \mathrm{Z} 202$ ) expresa 'laguna' en general, lo mismo que la variante libón, con aglutinación del artículo, que se recoge en $\mathrm{Hu} 200,201,202$; en $\mathrm{Hu} 200$ aparecen como sinónimos libón y otra voz dialectal gorgo 21 .

En gascón encontramos formas que se corresponden con las altoaragonesas: ioû, eoû, lhéoû 'lac' (Palay, 587).

Todas estas voces suponen una base prerromana *i b o n e, del mismo radical que el vasco ibai 'río' e ibar 'vega' (Azkue) ${ }^{22}$.

\section{3. 'TERRÓN DE TIERRA'}

Se registra la forma dialectal torroco, que se extiende por el norte y centro de Huesca y un pueblo de Teruel (Te 201), junto con las variantes torrueco (Hu 105, 202, 203, 204, 302, 405, Z 202), tarrueco (Hu 406), tarroc (Hu 402, 404, 408); es una voz que tiene correspondencias en gascón turròc, tarròc 'motte de terre' ${ }^{23}$ y en vasco tarroka 'terrón' (Azkue).

En algunos puntos hallamos como sinónimos este término y la voz dialectal tormo (Hu 102, 103, 104, 105, 106, 110, 206, 407), pero ésta no es exclusiva de este dominio lingüístico, ya que se documenta en otras zonas peninsulares ${ }^{24}$. A veces, entre ambas voces se establece una diferencia significativa basada en el sema 'tamaño': tormo / to. rrueco (Hu 107), torroco / tormo (Hu 112), que designan 'terrón de tierra de menor y mayor tamaño'.

La disposición o las determinadas características que tiene la masa

${ }^{21}$ Cfr. A. Badía, op. cit., p. 285: gorgo 'hoyo grande donde se detiene y estanca el agua de un rio'; cfr. además las correspondientes voces gasconas gurgo, gurg, gurga 'flaque boueuse', J. Séguy, Atlas linguistique et etnographique de la Gascogne. Paris, 1954-66, vol. III, carte $n .^{\circ} 783$; estos vocablos proceden del latín vulgar g ù r g u s, g ù r g a, modificación del clásico $g$ u $r g$ e $s$, - i $t$ i s 'lugar profundo en un río' (DCECH, III, pp. 174-5).

${ }_{22}$ Cfr. G. Rohlfs, op. cit., p. 53.

23 lbid., p. 71.

24 Es la forma más extendida por Navarra y La Rioja tal como aparece en el Atlas; también es la denominación habitual en Almeria y en diversas localidades granadinas, cfr. M. Alvar, Atlas lingütstico y etnografico de Andalucia, con la colaboración de A. Llorente y G. Salvador. Granada, 1961-73, mapa 893. 
de tierra no es un rasgo pertinente léxicamente en algunas ocasiones, y la voz torroco actualiza los significados de 'terrón de tierra' y 'terrón húmedo de forma alargada' en $\mathrm{Hu} \mathrm{200,} \mathrm{tarroc} \mathrm{en} \mathrm{Hu} 404$; en otros casos la distinción significativa se mantiene a través de los determinantes: torroc / torroc larguerul (Hu 205), torroco / torroco largudo (Hu 400), tarrueco / tarrueco largo (Hu 406), o a través de la especialización de cada uno de los términos dialectales: tormo / torroco (Hu 100), para expresar 'terrón de tierra' y 'terrón húmedo de forma alargada' respectivamente.

\section{4. 'TERRÓN HÚMEDO DE FORMA ALARGADA'}

La denominación metafórica chulla es la más extendida; se registra en Huesca, Zaragoza y Navarra, en esta última provincia hallamos la variante chula, con simplificación de la consonante palatal lateral.

Según señalan M. Alvar ${ }^{25}$ y V. García de Diego (DEEH) procede de una forma latina s ú i I l a 'carne de cerdo', con el paso de s->š-propio de las hablas pirenaicas. Para Corominas su étimo es el latín a $x$ u n g i a 'grasa de cerdo' que evolucionó a ensunya, según se atestigua en el catalán medieval, o *enxunya, posteriormente dio el catalán xulla (DCVB) y el aragonés chulla (Borao), por disimilación de palatales y por eliminación del seudoprefijo en-(DCECH, II, 404).

Su significado habitual es el de 'tajada de carne de cerdo', pero por un proceso de comparación metafórica basada, quizá, en la semejanza formal que se ha querido ver entre ambos elementos, ha tomado también este sentido. El mismo fenómeno ha ocurrido con la forma chuleta

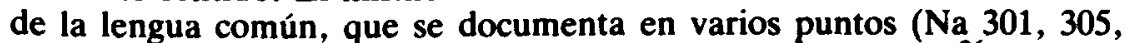
$402,403,405,600, Z 200,302,500,602$ ) con este significado ${ }^{26}$.

Otro vocablo dialectal es leba, que aparece en Huesca, con palatalización lleba en el norte y centro oriental y gleva en localidades fronterizas (Hu 602, Z 606, Te 202, 207, 401), que corresponde al catalán gleva 'terrón' (Diccionari, IV, 526). Su significado fundamental es el de 'terrón arrancado con hierba'; con el valor de 'terrón húmedo de forma alargada' sólo se recoge en unos pocos pueblos: leba (Hu 301, 603, Z 605, Te 201), gleva (Hu 602). Esta coincidencia en la denominación ha supuesto que en varios pueblos se hayan neutralizado los semas diferenciadores y la misma forma cubra los dos sentidos: leba en $\mathrm{Hu} \mathrm{302,} \mathrm{Z}$

${ }^{25} \mathrm{Cfr}$. M. Alvar, op. cit., p. 170.

${ }^{26}$ Comp. otras denominaciones metafóricas que se documentan en este ámbito lingüístico y que responden al mismo proceso comparativo: rebanada en el centro y sur de Teruel; tajada en la zona occidental de Zaragoza y en los pueblos de La Rioja y Navarra. 
601, lleba en $\mathrm{Hu}$ 402, 408, gleva en Te 207; en Hu 407 se mantiene la distinción a través del determinante: leba 'terrón húmedo alargado' / leba con hierba 'terrón arrancado con hierba'.

\section{5. 'Cueva'}

El término dialectal se conserva en muy pocos puntos: espelunga (Hu 101, 102), espolunga ( $Z$ 200) donde tiene el sentido específico de 'sitio escabroso', espluca (Hu 200), espluga (Hu 204, 400); en Hu 200 y $\mathrm{Hu} 204$ es voz polisémica que actualiza los significados de 'cueva' y 'saliente de una roca empleado como abrigo'; en estas mismas localidades los derivados correspondientes espluqueta (Hu 200), esplugueta (Hu 204) tienen el valor de 'covacha'.

Como topónimo local ${ }^{27}$ se mantiene Espelungueta en $\mathrm{Hu} 104$, pero para la denominación común se utiliza la palabra de la lengua general, cueva.

Estas formas están emparentadas con el catalán espluga 'cueva', 'caverna' (Diccionari, III, 658), y con el gascón espelugue, espeluque, espelungue 'caverna', 'gruta' (Palay, 455) 28.

Las voces del tipo espelunga derivan del latín s p e l u n c a 'caverna', mientras que las formas sin -n-: espluca, espluga proceden de una hipotética variante *s p e I u c a surgida, posiblemente, por un cruce entre el helenismo s p e l u n c a y la genuina palabra latina para designar 'caverna', s p e c u s ${ }^{29}$.

\section{CONSIDERACIONES FINALES}

Todas las formas analizadas se han mantenido arraigadas como términos autóctonos de este ámbito lingüístico, a pesar del empuje que ejercen las voces procedentes de la lengua general, bien porque estas palabras hacen referencia a paisajes y terrenos específicos de esta zona, o bien por la especial connotación de los vocablos; sin embargo, se hallan diferencias respecto a la extensión geográfica de cada uno de ellos,

${ }^{27}$ Cfr. M. Alvar, Toponimia del alto valle del río Aragón. Zaragoza, 1949, p. 52 y W.D. Elcock, "Toponimia del valle de Tena", en Archivo de Filologia Aragonesa. XIIXIII, 1961-62, pp. 315-6.

${ }^{2 \pi}$ Cfr. J. Séguy, op. cit., vol. III, carte n." 786.

${ }^{29}$ J. Corominas, «Espigueo de latín vulgar», en Topica Hesperica. Madrid, 1972. vol. I, pp. $359-362$ (s.v. espluca, espluga). 
ya que en unos casos han quedado reducidos a unos cuantos pueblos de los valles pirenaicos, y, en otros, por el contrario, ocupan no sólo la zona altoaragonesa, sino que se extienden a numerosas localidades de Navarra, Huesca, Zaragoza, incluso de Teruel, pasando además como préstamos a otras hablas peninsulares.

Aunque son numerosos los términos de origen prerromano que se registran, en su mayoría relacionados con el vasco, también son abundantes los vocablos de procedencia latina propios de este dominio, coincidentes en multitud de ocasiones con el gascón, y que difieren de los étimos preferidos por las hablas colindantes.

En muchas de estas palabras perviven rasgos fonéticos patrimoniales, como la posibilidad de diptongación de vocales cerradas (alcuerce); la palatalización de GL-inicial y la posterior desaparición de la oclusiva, rasgo característico de la zona ribagorzana (llera, lleba); el paso de /s- $/$ al fonema palatal $/ \hat{s}-/$ (chulla); la conservación de oclusivas sordas intervocálicas (ripazo, paco); la alternancia de los grupos /-rd-/, /-ĩ-/ (bardo, barro, barducal); el mantenimiento de /V procedente del grupo latino -T'L-(ruello) o -D'L-(ralla) en oposición al castellano, donde evolucionó al fonema velar $/ x /$; la conservación del grupo -NG'L(cingla, cinglo) frente a la diversidad de soluciones que presenta el castellano (ceño, cello, cincho \& c ì n g ŭ l u).

En ocasiones encontramos juntos el término normativo y el vocablo dialectal en una misma localidad; a veces, se establece una diferencia significativa entre ellos basada, en general, en el sema 'tamaño'; pero, en otros casos, no hay ninguna distinción, se trata simplemente de la preferencia por uno u otro según el contexto social en el que el hablante se exprese.

\section{ABREVIATURAS LEXICOGRÁFICAS}

Azkue. $=\mathrm{R} . \mathrm{M}^{\mathrm{a}}$ de Azkue, Diccionario vasco-español-francés (3. ${ }^{\mathrm{a}}$ ed. de la de Bilbao, 1905-6). Bilbao, 1984.

Borao = J. Borao, Diccionario de voces aragonesas (2. ${ }^{\mathrm{a}}$ ed.). Zaragoza, 1908.

D.C.V.B. = A. M. ${ }^{\text {a }}$ Alcover y F. de B. Moll, Diccionari català-valencià-balear (3." ed.). Palma de Mallorca, 1980.

D.C.E.C.H. = J. Corominas, Diccionario crítico etimológico castellano e hispánico, con la colaboración de J. A. Pascual. Madrid, Gredos, 1980 ss.

Diccionari $=\mathrm{J}$. Corominas, Diccionari etimològic $i$ complementari de la llengua catalana, amb la col-laboració de J. Gulsoy i Max Cahner. Barcelona, 1980 ss. 
D.E.E.H. = V. García de Diego, Diccionario etimológico español e hispánico (2. " ed.). Madrid, 1985.

D.R.A.E. = Real Academia Española, Diccionario de la lengua española (20. ${ }^{\mathrm{a}}$ ed.). Madrid, 1984.

Iribarren $=\mathrm{J}$. M..$^{\mathrm{a}}$ Iribarren, Vocabulario navarro (2. ${ }^{\mathrm{a}}$ ed.). Pamplona, 1984.

Palay $=$ S. Palay, Dictionnaire du béarnais et du gascon modernes (Bassin Aquitain). Paris, 1961.

Rohlfs = G. Rohlfs, Diccionario dialectal del Pirineo aragonés. Zaragoza, 1985. 